\title{
Coagulation and Fibrinolysis Parameters in Type 2 Diabetic Patients with and without Diabetic Vascular Complications
}

\author{
Cihangir Erem ${ }^{a, b}$ Arif Hacıhasanoğlu ${ }^{a, b}$ Şükrü Çelik ${ }^{c}$ Ercüment Ovalıa,d \\ H. Önder Ersöz ${ }^{a, b}$ Kubilay Ukinça,b Orhan Degere Münir Telatara,b \\ aDepartment of Internal Medicine, ${ }^{b}$ Division of Endocrinology and Metabolism and ${ }^{\mathrm{c} C}$ Cardiology, Departments of \\ ${ }^{\mathrm{d} H e m a t o l o g y}$ and eBiochemistry, Faculty of Medicine, Karadeniz Technical University, Trabzon, Turkey
}

\section{Key Words}

Type 2 diabetes · Coagulation · Fibrinolysis · Vascular complications · Lipid profile

\begin{abstract}
Objectives: To investigate the markers of endogenous coagulation/fibrinolysis and vascular endothelial cell function, and to assess the relationships between hemostatic parameters and diabetic vascular complications in type 2 diabetic patients. Materials and Methods: Coagulation and fibrinolysis parameters were measured in 92 type 2 diabetic patients ( 43 male, 49 female, mean age $50.1 \pm 13.4$ years) with $(n=44)$ and without $(n=48)$ vascular diabetic complications, and in 40 nondiabetic healthy subjects (20 male, 20 female, mean age $49.8 \pm$ 15.1 years). Common lipid parameters were also measured. Results: The plasma levels of fibrinogen, antithrombin III (AT III), plasminogen activator inhibitor-1 (PAl-1), von Willebrand factor (vWF) activity and prothrombin time were found to be significantly increased in the type 2 diabetic patients compared with the healthy subjects. Glycosylated hemoglobin Ic was inversely correlated with plasma protein $\mathrm{S}$ and activated prothrombin time. Protein $\mathrm{C}$ and $\mathrm{S}$ activities were positively correlated
\end{abstract}

\section{KARGER}

Fax +41613061234

E-Mail karger@karger.ch

www.karger.com

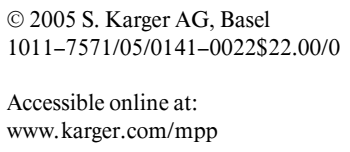

with plasma VWF activity, and were negatively correlated with plasma t-PA levels. vWF activity was negatively correlated with plasma t-PA levels. AT III levels were positively correlated with plasma total cholesterol levels, plasma low density lipoprotein cholesterol levels, plasma triglycerides and D-dimer levels. Plasma PAl-1 levels and factor $\mathrm{V}$ activity in diabetic patients with microvascular complications were significantly higher than those of the diabetic patients without microvascular complications. The plasma PAI- 1 and platelet count were increased in patients with diabetic retinopathy compared with the diabetic patients without retinopathy. Plasma PAI-1 levels and factor VII activity were significantly higher in the diabetic patients with nephropathy than in diabetic patients without nephropathy. Plasma concentrations of fibrinogen and PAI- 1 were significantly higher in the diabetic patients with neuropathy than the diabetic patients without neuropathy. Conclusions: The data demonstrated that patients with type 2 diabetes mellitus had a hypercoagulable state and hypofibrinolysis, thereby indicating that activation of coagulation with a reduced fibrinolytic activity may contribute to the increased risk of vascular disease in type 2 diabetic patients.

Copyright $@ 2005$ S. Karger AG, Basel
Prof. Dr. Cihangir Erem

K.T.Ü. Tip Fakültesi

Iç Hastalıkları Anabilim Dalı

TR-61080 Trabzon (Turkey)

Tel. +90 46237754 49, Fax +90 46232522 70, E-Mail cihangirerem@hotmail.com 


\section{Introduction}

Diabetes is associated with an increased risk of atherosclerosis, and coronary artery disease is a major cause of death in patients with diabetes $[1,2]$. Thrombosis is the cause of death in $80 \%$ of patients with diabetes. Of these deaths, $75 \%$ are due to cardiovascular complications, while the remaining $25 \%$ are due to cerebrovascular events and peripheral vascular complications [3].

It is well known that the vascular endothelium plays an essential role in the regulation of (local) hemostatic processes [4]. The major defense mechanisms that prevent inappropriate coagulation are found at the endothelial cell surface [5]. The endothelium itself is known to produce procoagulant factors such as von Willebrand factor (vWF), and anticoagulant factors, such as heparan sulfate and thrombomodulin [6, 7]. Endothelial dysfunction has also been shown to occur in type 2 diabetes. Multiple mechanisms are most likely involved; in fact, components of the insulin resistance syndrome may be central to the development of diabetic dysfunctional endothelium [8]. Various approaches (e.g. morphological, functional, and biologic) may be used to assess endothelial integrity. The plasma levels of biological markers, such as vWF, endothelin-1, and adhesion molecules in different proatherogenic conditions [9-11], may reflect endothelial function. Increased levels of vWF, a glycoprotein synthesized by endothelial cells in subjects with type 2 diabetes, are associated with vascular injury [12].

In diabetic patients, a procoagulant state is observed, which could contribute to the risk of catastrophic cardiovascular events. The plasma levels of many clotting factors including fibrinogen, factors VII, IX, XII, kallikrein and $\mathrm{vWF}$ are elevated in diabetes $[3,13,14]$. This hypercoagulable state could be caused by an imbalance between hemostatic factors in plasma, and the endothelial cell surface. So far, indirect evidence for a state favoring thrombin formation has been gathered, mainly using plasma parameters like fibrinogen and antithrombin activity or endothelium-dependent parameters like vWF [6,7].

The contribution of fibrinolytic system abnormalities to the development of diabetic micro- and macrovascular complications is still controversial [15]. In several studies activity of the fibrinolytic system in diabetics was found to be increased [16-18], decreased [19-21] or unchanged [22]. However, most studies indicate hypofibrinolysis leading to increased coagulation. Hypofibrinolysis may occur due to reduced expression of tissue plasminogen activator (t-PA) [7], which is the most important activator of plasminogen, or increased plasminogen activator in- hibitor-1 (PAI-1) [23-25]; PAI-1 is considered an independent factor of cardiovascular risk in type 2 diabetes mellitus.

The main purpose of this study was to investigate the markers of endogenous coagulation/fibrinolysis and of vascular endothelial cell function, and to assess the relationships between hemostatic parameters and diabetic vascular complications in patients with type 2 diabetes.

\section{Materials and Methods}

Ninety-two type 2 diabetic patients were included in the study (43 male, 49 female; mean age $50.1 \pm 13.4$ years). The patients were divided into two subgroups: patients with $(n=44)$ and without $(n=$ 48) vascular complications. The control group consisted of 40 age-, sex-, and body mass index (BMI)-matched nondiabetic healthy subjects ( 20 male, 20 female; mean age $49.8 \pm 15.1$ years $)$ with no family history of diabetes. Patients with retinopathy, neuropathy and/or nephropathy were considered to have microangiopathy. Retinopathy was diagnosed by an ophthalmologist on the basis of funduscopic examination. Microalbuminuria was defined as an albumin excretion rate of $3 \mathrm{mg} / \mathrm{mmol}$ creatinine in an early morning urine specimen on at least two consecutive occasions. The diagnosis of autonomic or somatic neuropathy was based on the presence of symptoms and/or signs of polyneuropathy and on the presence of at least two abnormal neurophysiological tests. The following neurophysiological tests were performed: ulnar and peroneal nerve motor conduction velocity. Moreover, to assess the presence of autonomic neuropathy the following cardiovascular autonomic tests were performed: Valsalva maneuver, deep breathing, lying to standing, orthostatic hypotension, and handgrip. Three patients were only on diet therapy, 62 on oral antidiabetic drug therapy, 22 on insulin therapy, and 5 on insulin plus oral antidiabetic combined therapy.

Subjects with clinical or laboratory signs of liver dysfunction, malignancy or a history of coagulation disorder were excluded from the study. Patients with manifest cardiovascular disease (history of myocardial infarction, angina pectoris, positive electrocardiographic changes, cerebrovascular accident and/or peripheral vascular disease) were also excluded. Another group that was excluded consisted of patients receiving medications that could affect the coagulationfibrinolytic system and lipid metabolism, such as anticoagulants and antiplatelet agents or oral contraceptives, hormonal replacement therapy, and hypolipidemic drugs.

Blood was collected in the morning between 8.00 and 9.00 a.m. after an overnight fast. Glucose levels were determined by the glucose oxidase method in serum. Glycosylated hemoglobin $\left(\mathrm{Hb} \mathrm{A}_{1 \mathrm{c}}\right)$ value (specific for $\mathrm{Hb} \mathrm{A}_{1 \mathrm{c}}$ ) was assayed in both diabetic and control groups with the latex immunoagglutination inhibition method (DCA 2000, Ames, Miles Inc., code 5036A). The samples for $\mathrm{Hb} \mathrm{A}_{1 \mathrm{c}}$ were obtained by finger prick taking approximately $1 \mu \mathrm{l}$ of capillary blood. Serum total cholesterol was measured using a cholesterol oxidase enzymatic method; triglycerides by a glycerol oxidase enzymatic method; high density lipoprotein cholesterol (HDL-C) by a cholesterol oxidase enzymatic method in supernatant after precipitation of other lipoproteins with phosphotungstic acid- $\mathrm{MgCl}_{2}$. These routine analyses were carried out by Autoanalyzer (Technicon AXON). Low 
Table 1. Clinical and biological parameters of controls and patients with type 2 diabetes

\begin{tabular}{|c|c|c|c|}
\hline & Controls & Diabetic patients & $\mathrm{p}$ \\
\hline \multicolumn{4}{|l|}{ Demographics } \\
\hline Subjects & 40 & 92 & - \\
\hline Age, years & $49.8 \pm 15.1$ & $50.9 \pm 13.4$ & NS \\
\hline Gender, F/M & $20 / 20$ & $49 / 43$ & - \\
\hline Duration of diabetes, years & - & $8.8 \pm 7.0$ & - \\
\hline $\mathrm{BMI}, \mathrm{kg} / \mathrm{m}^{2}$ & $2.4 \pm 7.3$ & $28.4 \pm 6.2$ & NS \\
\hline Systolic blood pressure, $\mathrm{mm} \mathrm{Hg}$ & $126.3 \pm 16.8$ & $138.7 \pm 25.1$ & $<0.05$ \\
\hline Diastolic blood pressure, $\mathrm{mm} \mathrm{Hg}$ & $75.4 \pm 13.5$ & $86.5 \pm 14.7$ & $<0.05$ \\
\hline \multicolumn{4}{|l|}{ Biochemical parameters } \\
\hline Fasting blood glucose, mg/dl & $74.5 \pm 6.2$ & $220.6 \pm 81.6$ & $<0.0001$ \\
\hline $\mathrm{Hb} \mathrm{A}, \%$ & $5.4 \pm 0.8$ & $10.0 \pm 2.4$ & $<0.0001$ \\
\hline Platelet count per $\mu \mathrm{l}$ & $233.8 \pm 36.7$ & $244.0 \pm 57.6$ & NS \\
\hline Total cholesterol, mg/dl & $157.5 \pm 32.1$ & $218.4 \pm 69.3$ & $<0.01$ \\
\hline Triglycerides, mg/dl & $111.2 \pm 68.6$ & $186.4 \pm 130.0$ & NS \\
\hline LDL-C, mg/dl & $91.6 \pm 29.9$ & $141.5 \pm 58.9$ & $<0.01$ \\
\hline HDL-C, mg/dl & $43.4 \pm 5.2$ & $53.1 \pm 10.2$ & NS \\
\hline \multicolumn{4}{|l|}{ Common coagulation studies } \\
\hline Prothrombin time, $\mathrm{s}$ & $11.1 \pm 0.4$ & $12.0 \pm 1.2$ & $<0.05$ \\
\hline aPTT & $31.2 \pm 3.4$ & $30.7 \pm 4.5$ & NS \\
\hline INR & $0.90 \pm 0.16$ & $1.45 \pm 2.09$ & NS \\
\hline D-dimer & $280.0 \pm 98.9$ & $263.9 \pm 139.4$ & NS \\
\hline Fibrinogen, mg/dl & $253.4 \pm 4.1$ & $291.2 \pm 77.1$ & $<0.05$ \\
\hline \multicolumn{4}{|l|}{ Special coagulation studies } \\
\hline Factor V, \% & $85.3 \pm 16.9$ & $85.8 \pm 29.5$ & NS \\
\hline Factor VII, \% & $89.6 \pm 12.8$ & $82.1 \pm 16.9$ & NS \\
\hline Factor VIII, \% & $123.3 \pm 31,7$ & $128.3 \pm 34.8$ & NS \\
\hline Factor $\mathrm{X}, \%$ & $92.8 \pm 20.2$ & $94.0 \pm 28.8$ & NS \\
\hline AT III Ag, mg/dl & $24.5 \pm 2.7$ & $28.5 \pm 4.2$ & $<0.0001$ \\
\hline Protein $C, \%$ & $109.3 \pm 29.2$ & $115.1 \pm 31.8$ & NS \\
\hline Protein S, \% & $118.9 \pm 32.8$ & $112.6 \pm 29.6$ & NS \\
\hline vWF activity, \% & $98.7 \pm 16.8$ & $114.5 \pm 27.5$ & $<0.05$ \\
\hline t-PA Ag, ng/ml & $11.8 \pm 2.9$ & $16.2 \pm 8.8$ & NS \\
\hline PAI- $1 \mathrm{Ag}, \mathrm{ng} / \mathrm{ml}$ & $21.4 \pm 5.8$ & $44.6 \pm 10.6$ & $<0.0001$ \\
\hline
\end{tabular}

INR = International normalized ratio. density lipoprotein cholesterol (LDL-C) was calculated by the Friedewald's formulae.

For coagulation and fibrinolysis, a venous blood sample $(9 \mathrm{ml})$ was collected into Vacutainer tubes (Becton Dickinson, Mountain View, Calif., USA) containing $0.129 \mathrm{~mol} / 1$ trisodium citrate $(1 \mathrm{vol})$. Platelet-poor plasma was obtained by centrifugation at $3,500 \mathrm{~g}$ at $10^{\circ} \mathrm{C}$ for $20 \mathrm{~min}$. Platelet count, prothrombin time, activated partial thromboplastin time (aPTT), fibrinogen, antithrombin III (AT III), factors V, VII, VIII, and X measurements were performed promptly. Aliquots of plasma were transferred into plastic tubes without delay and frozen at $-80^{\circ} \mathrm{C}$ until assays for determination of $\mathrm{vWF}$, protein $\mathrm{C}$, protein S, t-PA, and PAI-1. Platelet counts were measured with an automatic cell counter (Coulter Micro Diff II). Prothrombin time and aPTT were determined with coagulometer, using commercial kits of Diagnostica Stago. Fibrinogen was determined using a nephelometric assay by commercial kits for fibrinogen (Cat No. OSCA 09,
Dade Behring Marburg GmbH, Germany). Factor V, VII, VIII and X activities were measured with coagulometer (Diagnostica Stago) using commercial kits of Diagnostica Stago. AT III assay was performed with spectrophotometric method (Behring turbitimer, Turbiquant, AT III, Dade Behring). Normal ranges are $200-400 \mathrm{mg} / \mathrm{dl}$ for fibrinogen, $50-150 \%$ for factors V, VII, VIII and X. Protein C and protein $\mathrm{S}$ activity assays were performed with ELISA method using commercial kits of Biopool International. The vWF activity was determined by ELISA method using commercial kits of Imtec Immundiagnostica $\mathrm{GmbH}$. t-PA and PAI-1 assays were performed with ELISA using commercial kits of American Diagnostica. According to the manufacturer, normal ranges are $22-39 \mathrm{mg} / \mathrm{dl}$ for AT III, $70-150 \%$ for vWF, $72-160 \%$ for protein C activity, $60-150 \%$ for protein S activity, $1-20 \mathrm{ng} / \mathrm{ml}$ for t-PA Ag, and $20-44 \mathrm{ng} / \mathrm{ml}$ for PAI-1 Ag. 
Table 2. Clinical and laboratory characteristics in diabetic patients with and without microvascular complications

\begin{tabular}{|c|c|c|c|}
\hline & \multicolumn{2}{|l|}{ Diabetic patients } & \multirow[t]{2}{*}{$\mathrm{p}$} \\
\hline & with complications & without complications & \\
\hline \multicolumn{4}{|l|}{ Demographics } \\
\hline Subjects & 44 & 48 & - \\
\hline Age, years & $54.84 \pm 10.88$ & $46.94 \pm 15.30$ & $<0.01$ \\
\hline Gender, F/M & $24 / 20$ & $25 / 23$ & - \\
\hline Duration of diabetes, years & $11.27 \pm 6.94$ & $6.3 \pm 5.65$ & $<0.001$ \\
\hline $\mathrm{BMI}, \mathrm{kg} / \mathrm{m}^{2}$ & $28.58 \pm 5.52$ & $28.95 \pm 6.50$ & NS \\
\hline Systolic blood pressure, $\mathrm{mm} \mathrm{Hg}$ & $141.13 \pm 28.66$ & $136.11 \pm 23.95$ & NS \\
\hline Diastolic blood pressure, $\mathrm{mm} \mathrm{Hg}$ & $86.13 \pm 15.30$ & $85.74 \pm 13.28$ & NS \\
\hline \multicolumn{4}{|l|}{ Biochemical parameters } \\
\hline Fasting blood glucose, $\mathrm{mg} / \mathrm{dl}$ & $251.98 \pm 83.18$ & $191.66 \pm 67.02$ & $<0.001$ \\
\hline $\mathrm{Hb} \mathrm{A}_{1 \mathrm{c}}, \%$ & $10.47 \pm 2.17$ & $9.26 \pm 2.29$ & $<0.05$ \\
\hline Total cholesterol, mg/dl & $223.73 \pm 63.72$ & $209.12 \pm 61.65$ & NS \\
\hline Triglycerides, mg/dl & $190.3 \pm 134.9$ & $189.9 \pm 113.4$ & NS \\
\hline $\mathrm{LDL}-\mathrm{C}, \mathrm{mg} / \mathrm{dl}$ & $152.13 \pm 57.98$ & $121.74 \pm 42.30$ & $<0.05$ \\
\hline HDL-C, mg/dl & $43.75 \pm 10.62$ & $43.47 \pm 10.62$ & NS \\
\hline \multicolumn{4}{|l|}{ Common coagulation studies } \\
\hline Prothrombin time, $\mathrm{s}$ & $11.87 \pm 1.20$ & $11.92 \pm 1.20$ & NS \\
\hline aPTT & $30.56 \pm 4.45$ & $31.15 \pm 4.84$ & NS \\
\hline INR & $1.45 \pm 0.90$ & $0.90 \pm 0.16$ & NS \\
\hline D-dimer & $263.90 \pm 139.48$ & $280.00 \pm 98.99$ & NS \\
\hline Fibrinogen, $\mathrm{mg} / \mathrm{dl}$ & $285.38 \pm 43.64$ & $268.80 \pm 16.61$ & NS \\
\hline \multicolumn{4}{|l|}{ Special coagulation studies } \\
\hline Factor V, \% & $90.45 \pm 27.48$ & $73.54 \pm 20.43$ & $<0.05$ \\
\hline Factor VII, \% & $82.72 \pm 18.88$ & $81.76 \pm 16.61$ & NS \\
\hline Factor VIII, \% & $130.66 \pm 34.58$ & $129.44 \pm 38.15$ & NS \\
\hline Factor $\mathrm{X}, \%$ & $106.48 \pm 32.66$ & $74.40 \pm 11.65$ & NS \\
\hline AT III Ag, mg/dl & $28.29 \pm 4.69$ & $29.05 \pm 3.96$ & NS \\
\hline Protein $C, \%$ & $115.13 \pm 31.81$ & $109.27 \pm 29.23$ & NS \\
\hline Protein S, \% & $112.63 \pm 29.55$ & $115.86 \pm 32.82$ & NS \\
\hline vWF activity, $\%$ & $118.90 \pm 26.76$ & $111.36 \pm 26.34$ & NS \\
\hline t-PA Ag, ng/ml & $16.05 \pm 10.50$ & $14.93 \pm 6.07$ & NS \\
\hline PAI-1 Ag, $\mathrm{ng} / \mathrm{ml}$ & $47.55 \pm 9.45$ & $40.82 \pm 11.50$ & $<0.05$ \\
\hline
\end{tabular}

INR = International normalized ratio.
Statistical analysis was performed by Student's t test, MannWhitney U test, and multiple regression analysis. Results are presented as the mean \pm SD. The calculations were performed using SPSS for Windows version 10 (SPSS, Inc., Chicago, Ill., USA).

\section{Results}

The clinical characteristics of all subjects, including their plasma lipid profile and markers of the coagulation/ fibrinolytic system, are summarized in table 1 . No difference was found in age or BMI between the diabetic patients and control subjects. The levels of plasma AT-III, fibrinogen, PAI-1, and vWF activity were significantly increased and prothrombin time was longer in the type 2 diabetic patients than the healthy subjects. Coagulation factors (V, VII, VIII and X), platelet count, aPTT, Ddimer, t-PA, protein $\mathrm{C}$, and protein $\mathrm{S}$ did not differ between patients and control subjects. Duration of diabetes, plasma PAI-1 levels and factor V activity in type 2 diabetic patients with diabetic microvascular complications were significantly higher than those of the type 2 diabetic patients without diabetic microvascular complications (table 2). Also, the plasma PAI-1 levels and platelet count were increased in patients with diabetic retinopathy compared with the diabetic patients without retinopathy (table 3). Plasma PAI-1 levels and factor VII activity 
Table 3. Clinical and laboratory characteristics in diabetic patients with and without retinopathy
With retinopathy Without retinopathy $p$

\begin{tabular}{|c|c|c|c|}
\hline \multicolumn{4}{|l|}{ Demographics } \\
\hline Subjects & 33 & 59 & - \\
\hline Age, years & $56.94 \pm 10.77$ & $46.21 \pm 13.66$ & $<0.0001$ \\
\hline Duration of diabetes, years & $11.7 \pm 7.38$ & $6.92 \pm 5.42$ & $<0.01$ \\
\hline $\mathrm{BMI}, \mathrm{kg} / \mathrm{m}^{2}$ & $27.40 \pm 4.33$ & $29.74 \pm 7.19$ & NS \\
\hline Systolic blood pressure, $\mathrm{mm} \mathrm{Hg}$ & $153.62 \pm 28.78$ & $130.68 \pm 18.79$ & $<0.0001$ \\
\hline Diastolic blood pressure, $\mathrm{mm} \mathrm{Hg}$ & $90.00 \pm 16.15$ & $85.00 \pm 13.69$ & NS \\
\hline \multicolumn{4}{|l|}{ Biochemical parameters } \\
\hline Fasting blood glucose, $\mathrm{mg} / \mathrm{dl}$ & $261.67 \pm 86.43$ & $195.54 \pm 66.09$ & $<0.0001$ \\
\hline $\mathrm{Hb} \mathrm{A}=\%$ & $10.64 \pm 2.09$ & $9.21 \pm 2.29$ & $<0.05$ \\
\hline Platelet count per $\mu \mathrm{l}$ & $262.90 \pm 56.79$ & $231.5 \pm 56.1$ & $<0.05$ \\
\hline Total cholesterol, mg/dl & $219.84 \pm 68.13$ & $220.16 \pm 58.46$ & NS \\
\hline Triglycerides, $\mathrm{mg} / \mathrm{dl}$ & $170.09 \pm 116.78$ & $207.92 \pm 136.38$ & NS \\
\hline LDL-C, $\mathrm{mg} / \mathrm{dl}$ & $146.96 \pm 60.21$ & $143.90 \pm 45.08$ & NS \\
\hline HDL-C, mg/dl & $43.71 \pm 11.57$ & $53.95 \pm 11.67$ & NS \\
\hline \multicolumn{4}{|l|}{ Common coagulation studies } \\
\hline Prothrombin time, $\mathrm{s}$ & $11.79 \pm 1.18$ & $11.99 \pm 1.23$ & NS \\
\hline aPTT & $30.91 \pm 4.72$ & $30.84 \pm 4.68$ & NS \\
\hline INR & $1.54 \pm 2.35$ & $0.93 \pm 0.19$ & NS \\
\hline D-dimer & $271.00 \pm 146.01$ & $270.00 \pm 95.96$ & NS \\
\hline Fibrinogen, $\mathrm{mg} / \mathrm{dl}$ & $282.53 \pm 35.09$ & $268.44 \pm 15.24$ & NS \\
\hline \multicolumn{4}{|l|}{ Special coagulation studies } \\
\hline Factor V, \% & $89.22 \pm 28.79$ & $78.22 \pm 21.54$ & NS \\
\hline Factor VII, \% & $85.51 \pm 19.42$ & $80.24 \pm 16.15$ & NS \\
\hline Factor VIII, \% & $132.16 \pm 37.23$ & $130.13 \pm 35.56$ & NS \\
\hline Factor X, \% & $106.48 \pm 32.66$ & $74.40 \pm 11.65$ & NS \\
\hline AT III Ag, mg/dl & $27.71 \pm 4.64$ & $29.60 \pm 3.86$ & NS \\
\hline Protein C, \% & $110.00 \pm 30.81$ & $114.84 \pm 31.12$ & NS \\
\hline Protein S, \% & $112.13 \pm 30.11$ & $119.04 \pm 29.66$ & NS \\
\hline vWF activity, $\%$ & $114.79 \pm 26.95$ & $119.16 \pm 26.88$ & NS \\
\hline $\mathrm{t}-\mathrm{PA} \mathrm{Ag}, \mathrm{ng} / \mathrm{ml}$ & $16.15 \pm 10.33$ & $15.28 \pm 7.97$ & NS \\
\hline PAI-1 Ag, ng/ml & $48.26 \pm 8.24$ & $40.90 \pm 12.05$ & $<0.05$ \\
\hline
\end{tabular}

INR = International normalized ratio. were significantly higher in the diabetic patients with nephropathy than in diabetic patients without nephropathy (table 4). Plasma concentrations of fibrinogen and PAI-1 were significantly higher in the diabetic patients with neuropathy than the diabetic patients without neuropathy (table 5).

In the study group, $\mathrm{Hb} \mathrm{A}_{1 \mathrm{c}}$ was negatively correlated with plasma protein $\mathrm{S}$ activity $(\mathrm{r}=-0.30, \mathrm{p}<0.05)$ and $\operatorname{aPTT}(\mathrm{r}=-0.27, \mathrm{p}<0.05)$. Protein $\mathrm{C}$ and $\mathrm{S}$ activities were positively correlated with plasma $\mathrm{vWF}$ activity $(\mathrm{r}=0.65$, $\mathrm{p}<0.0001 ; \mathrm{r}=0.57, \mathrm{p}<0.0001$, respectively) and were negatively correlated with plasma t-PA levels $(r=-0.45$, $\mathrm{p}<0.0001$ and $\mathrm{r}=0.43, \mathrm{p}<0.001$, respectively). The vWF activity was negatively correlated with plasma t-PA levels $(r=-0.29, p<0.05)$. AT III levels were positively correlated with plasma total cholesterol levels $(r=0.32$, $\mathrm{p}<0.05)$, plasma LDL-C levels $(\mathrm{r}=0.42, \mathrm{p}<0.05)$, plasma triglycerides $(r=0.33, p<0.05)$ and $D$-dimer levels $(\mathrm{r}=0.64, \mathrm{p}<0.01)$.

\section{Discussion}

Enhanced activation of the clotting system has been previously reported in patients with type 1 or type 2 diabetes [26]. This activation of blood coagulation has recently been implicated as an important contributing factor for the occurrence of vascular complications in dia- 
Table 4. Clinical and laboratory characteristics in diabetic patients with and without nephropathy
With nephropathy Without nephropathy $\mathrm{p}$

\begin{tabular}{|c|c|c|c|}
\hline \multicolumn{4}{|l|}{ Demographics } \\
\hline Subjects & 16 & 76 & - \\
\hline Age, years & $58.44 \pm 8.08$ & $48.96 \pm 14.07$ & $<0.05$ \\
\hline Duration of diabetes, years & $15.14 \pm 8.34$ & $7.13 \pm 5.45$ & $<0.0001$ \\
\hline $\mathrm{BMI}, \mathrm{kg} / \mathrm{m}^{2}$ & $28.19 \pm 4.99$ & $28.76 \pm 6.50$ & NS \\
\hline Systolic blood pressure, $\mathrm{mm} \mathrm{Hg}$ & $163.33 \pm 24.69$ & $132.69 \pm 22.85$ & $<0.0001$ \\
\hline Diastolic blood pressure, $\mathrm{mm} \mathrm{Hg}$ & $91.00 \pm 17.95$ & $85.96 \pm 13.14$ & NS \\
\hline \multicolumn{4}{|l|}{ Biochemical parameters } \\
\hline Fasting blood glucose, $\mathrm{mg} / \mathrm{dl}$ & $255.38 \pm 67.03$ & $209.65 \pm 81.67$ & $<0.05$ \\
\hline $\mathrm{Hb} \mathrm{A}_{1 \mathrm{c}}, \%$ & $10.60 \pm 2.48$ & $9.53 \pm 2.22$ & NS \\
\hline Platelet count per $\mu \mathrm{l}$ & $273.56 \pm 72.71$ & $236.47 \pm 53.53$ & NS \\
\hline Total cholesterol, mg/dl & $232.88 \pm 76.53$ & $215.02 \pm 60.23$ & NS \\
\hline Triglycerides, mg/dl & $180.63 \pm 121.21$ & $188.70 \pm 130.10$ & NS \\
\hline $\mathrm{LDL}-\mathrm{C}, \mathrm{mg} / \mathrm{dl}$ & $173.25 \pm 75.38$ & $136.55 \pm 49.50$ & NS \\
\hline HDL-C, mg/dl & $37.50 \pm 5.73$ & $45.64 \pm 13.88$ & NS \\
\hline \multicolumn{4}{|l|}{ Common coagulation studies } \\
\hline Prothrombin time, s & $11.52 \pm 1.11$ & $11.99 \pm 1.23$ & NS \\
\hline aPTT & $32.05 \pm 6.35$ & $30.57 \pm 4.11$ & NS \\
\hline INR & $2.16 \pm 3.35$ & $0.92 \pm 0.16$ & NS \\
\hline D-dimer & $284.14 \pm 165.26$ & $261.00 \pm 87.49$ & NS \\
\hline Fibrinogen, mg/dl & $272.67 \pm 10.15$ & $275.65 \pm 30.42$ & NS \\
\hline \multicolumn{4}{|l|}{ Special coagulation studies } \\
\hline Factor V, \%, & $90.46 \pm 18.80$ & $81.21 \pm 28.00$ & NS \\
\hline Factor VII, \% & $94.72 \pm 21.31$ & $78.25 \pm 14.79$ & $<0.01$ \\
\hline FactorVIII, \% & $137.15 \pm 34.86$ & $127.28 \pm 36.55$ & NS \\
\hline Factor $\mathrm{X}, \%$ & $102.97 \pm 28.37$ & $89.66 \pm 32.22$ & NS \\
\hline AT III Ag, mg/dl & $28.46 \pm 4.98$ & $28.80 \pm 4.06$ & NS \\
\hline Protein $\mathrm{C}, \%$ & $96.18 \pm 20.38$ & $118.61 \pm 31.07$ & NS \\
\hline Protein S, \% & $112.00 \pm 29.24$ & $115.58 \pm 32.20$ & NS \\
\hline vWF activity, \% & $113.17 \pm 20.07$ & $119.47 \pm 26.16$ & NS \\
\hline $\mathrm{t}-\mathrm{PA} \mathrm{Ag}, \mathrm{ng} / \mathrm{ml}$ & $17.26 \pm 10.98$ & $14.24 \pm 7.98$ & NS \\
\hline PAI-1 Ag, ng/ml & $51.27 \pm 6.74$ & $43.28 \pm 11.15$ & $<0.05$ \\
\hline
\end{tabular}

INR = International normalized ratio betes $[27,28]$. In large epidemiological studies, the procoagulant factors (fibrinogen and factor VII) have been described as independent predictors of cardiovascular events in diabetic and nondiabetic subjects. In the present study, the plasma levels of fibrinogen were found to be significantly higher in diabetic patients than in those of the control subjects. Equally, Asakawa et al. [29] had shown that fibrinogen level was significantly higher in diabetic patients with retinopathy or nephropathy than in patients without these complications. The detection of microalbuminuria, another well-known independent indicator of much greater risk of premature death from cardiovascular complications, has been demonstrated to correlate significantly with various hemostatic variables [3,
26, 27, 30, 31]. Accordingly, some coagulation factors did not differ between the diabetic patients and control subjects. Increased factor $\mathrm{V}$ activity in diabetic patients with vascular complications and increased factor VII activity in patients with diabetic nephropathy were found. Our findings for fibrinogen are in accordance with the observations of Kvasnicka et al. [14], Ostermann et al. [6], Asakawa et al. [29] and $\mathrm{Hu}$ et al. [32]. However, the fibrinogen levels did not differ between the diabetic patients with and without vascular complications except for the patients with diabetic neuropathy. Asakawa et al. [29] showed that the duration of diabetes was significantly longer in patients with any microangiopathy than in patients without it, similar to our data. 
Table 5. Clinical and laboratory characteristics in diabetic patients with and without neuropathy

\begin{tabular}{|c|c|c|c|}
\hline & With neuropathy & Without neuropathy & $\mathrm{p}$ \\
\hline \multicolumn{4}{|l|}{ Demographics } \\
\hline Subjects & 59 & 33 & - \\
\hline Age, years & $54.24 \pm 11.68$ & $48.35 \pm 14.12$ & $<0.05$ \\
\hline Duration of diabetes, years & $12.71 \pm 6.97$ & $6.11 \pm 5.36$ & $<0.0001$ \\
\hline $\mathrm{BMI}, \mathrm{kg} / \mathrm{m}^{2}$ & $28.03 \pm 4.05$ & $29.05 \pm 7.17$ & NS \\
\hline Systolic blood pressure, $\mathrm{mm} \mathrm{Hg}$ & $147.50 \pm 27.38$ & $134.11 \pm 22.77$ & $<0.05$ \\
\hline Diastolic blood pressure, $\mathrm{mm} \mathrm{Hg}$ & $87.33 \pm 16.28$ & $86.78 \pm 12.39$ & NS \\
\hline \multicolumn{4}{|l|}{ Biochemical parameters } \\
\hline Fasting blood glucose, mg/dl & $208.02 \pm 83.94$ & $242.79 \pm 75.16$ & $<0.05$ \\
\hline $\mathrm{Hb} \mathrm{A}=\%$ & $10.23 \pm 2.17$ & $9.62 \pm 2.42$ & NS \\
\hline Total cholesterol, mg/dl & $220.52 \pm 65.63$ & $223.46 \pm 73.27$ & NS \\
\hline Triglycerides, mg/dl & $192.94 \pm 140.06$ & $194.0 \pm 118.83$ & NS \\
\hline LDL-C, mg/dl & $147.55 \pm 62.02$ & $143.61 \pm 60.0$ & NS \\
\hline HDL-C, mg/dl & $44.3 \pm 10.2$ & $41.87 \pm 10.35$ & NS \\
\hline \multicolumn{4}{|l|}{ Common coagulation studies } \\
\hline Prothrombin time, $\mathrm{s}$ & $11.98 \pm 1.21$ & $11.83 \pm 1.21$ & NS \\
\hline aPTT & $31.19 \pm 4.80$ & $30.35 \pm 4.23$ & NS \\
\hline INR & $1.45 \pm 2.09$ & $0.91 \pm 0.16$ & NS \\
\hline D-dimer & $271.00 \pm 146.01$ & $270.00 \pm 95.92$ & NS \\
\hline Fibrinogen, mg/dl & $294.44 \pm 51.23$ & $270.80 \pm 19.98$ & $<0.05$ \\
\hline \multicolumn{4}{|l|}{ Special coagulation studies } \\
\hline Factor V, \% & $93.60 \pm 30.64$ & $79.83 \pm 29.31$ & NS \\
\hline Factor VII, \% & $86.94 \pm 20.54$ & $79.40 \pm 15.30$ & NS \\
\hline Factor VIII, \% & $139.28 \pm 34.70$ & $124.75 \pm 35.82$ & NS \\
\hline Factor $\mathrm{X}, \%$ & $106.48 \pm 32.66$ & $74.4 \pm 11.65$ & NS \\
\hline AT III Ag, mg/dl & $28.02 \pm 5.03$ & $29.01 \pm 3.91$ & NS \\
\hline Protein $\mathrm{C}, \%$ & $111.84 \pm 33.11$ & $114.09 \pm 29.91$ & NS \\
\hline Protein S, \% & $110.84 \pm 32.39$ & $115.29 \pm 29.33$ & NS \\
\hline vWF activity, \% & $111.25 \pm 24.12$ & $118.03 \pm 28.99$ & NS \\
\hline t-PA Ag, ng/ml & $14.37 \pm 9.05$ & $16.24 \pm 8.55$ & NS \\
\hline PAI-1 Ag, ng/ml & $50.79 \pm 6.66$ & $41.39 \pm 11.11$ & $<0.001$ \\
\hline
\end{tabular}

INR = International normalized ratio.
The pathogenetic mechanism of the clotting activation in diabetes is not completely clear. Perturbance of components of the anticoagulant system associated with hyperglycemia may play an important role as exemplified in hyperglycemia inducing depressed biological activity of the anticoagulant protein AT-III in diabetic and nondiabetic subjects [33]. Structural modification due to nonenzymatic glycation was suggested as the causative factor of this AT-III dysfunction [34]. In various studies, it was reported that plasma levels of AT-III were decreased [35] or unchanged [32]. In these studies, increased thrombinantithrombin complex was found. In the present study we demonstrated an increased AT-III level in diabetic patients. Because AT-III is a coagulation inhibitor, this increase could also be a compensatory reaction to a procoagulant state, as can be found in diabetes.

Abnormalities of the anticoagulant protein $C$ pathway may also cause hypercoagulability in diabetic patients. The plasma antigen levels of protein $\mathrm{C}$ and of its cofactor protein $\mathrm{S}$ were found to be either decreased or increased in various reports depending on the type of diabetes. While reduced plasma levels of protein $\mathrm{C}$ antigen have been described in type 1 diabetic patients, they were found to be elevated in those with type 2 diabetes [26, 28, 36]. The activities of protein $\mathrm{C}$ and $\mathrm{S}$ did not change in our diabetic patients. Hyperglycemia has been considered to be the causative factor of the abnormalities of the protein $\mathrm{C}$ anticoagulant pathway. In our type 2 diabetic 
patients, we found a significant negative correlation between $\mathrm{Hb} \mathrm{A}_{1 \mathrm{c}}$ and protein S activity.

Endothelial dysfunction has been shown to occur in type 2 diabetes $[1,11]$. The $v W F, t-P A$ and PAI- 1 are among the important endothelium-derived proteins. Although a gold standard for endothelial dysfunction is not available, high plasma vWF and/or PAI-1 have been widely considered as markers of general endothelial dysfunction in serial diseases [37-39]. Increased levels of vWF in subjects with type 2 diabetes are associated with macrovascular mortality [12]. Several studies have shown that levels of vWF are increased in both type 1 and type 2 diabetic patients $[3,32,40]$ and that glycemic control normalizes vWF levels in diabetic patients [41]. In our study, we found an increased vWF activity in type 2 diabetics in comparison with the healthy controls. However, the mean values of both groups were within normal ranges of our laboratory. vWF activity did not differ between the diabetic patients with and without vascular complications.

Previous studies of the fibrinolytic system in diabetes have provided conflicting results; type 2 diabetes is often associated with profound depression of fibrinolysis asso- ciated with high levels of PAI-1 and t-PA [23, 24, 42, 43], while Aso et al. [44] reported that both coagulation and fibrinolysis are enhanced concomitantly in patients with type 2 diabetes mellitus. Our findings of increased plasma levels of PAI-1 and decreased (but not significant) t-PA are consistent with hypofibrinolysis. We also found a significant relationships between PAI-1 and retinopathy, nephropathy and neuropathy. Correlation between ATIII and LDL-C may be interpreted as a tendency to thrombosis with cardiovascular disease for diabetic patients.

\section{Conclusion}

The results showed that type 2 diabetic patients have high fibrinogen, AT-III, PAI-1 levels and vWF activity. Our data further demonstrated that patients with type 2 diabetes mellitus had hypercoagulable state and hypofibrinolysis thereby indicating that the activation of coagulation and reduced fibrinolytic activity may contribute to the increased risk of vascular disease in type 2 diabetic patients.

\section{References}

$>1$ Seligman BGS, Biolo A, Polanczyk C, Gross JL, Clausell N: Increased plasma levels of endothelin 1 and von Willebrand factor in patients with type 2 diabetes and dyslipidemia. Diabetes Care 2000;23:1395-1400.

$\checkmark 2$ Erem C, Deger O, Bostan M, Orem A, Sonmez M, Ulusoy S, Telatar M: Plasma lipoprotein (a) levels in Turkish NIDDM patients with and without vascular diabetic complications. Acta Cardiol 1999;54:203-207.

$\checkmark 3$ Carr ME: Diabetes mellitus: A hypercoagulable state. J Diabetes Complications 2001;15:4454.

$\checkmark 4$ Stern DM, Esposito C, Gerlach M, Ryan J, Handley D, Nawroth P: Endothelium and regulation of coagulation. Diabetes Care 1991; 14(suppl I):160-166.

$\checkmark 5$ Bourin M, Lindahl U: Glycosaminoglycans and the regulation of blood coagulation. Biochem J 1993;289:313-330.

6 Ostermann H, van de Loo J: Factors of the hemostatic system in diabetic patients: A survey of controlled studies. Haemostasis 1986; 16:386-416.

7 Carmassi F, Morale M, Puccetti R, De Negri F, Monzani F, Navakesi R, Mariani G: Coagulation and fibrinolytic system impairment in insulin dependent diabetes mellitus. Thromb Res 1992;67:643-654.

-8 Fagan TC, Deedwania PC: The cardiovascular dysmetabolic syndrome. Am J Med 1998; 105(suppl 1A):77S-82S
$>9$ Morise T, Takeuchi Y, Kawano M, Koni I, Takeda R: Increased plasma levels of immunoreactive endothelin and von Willebrand factor in NIDDM patients. Diabetes Care 1995;18: 87-89.

10 Clausell N, Prado KF, Ribeiro JP: Increased plasma levels of soluble vascular adhesion molecule-1 in patients with chest pain and angiographically normal coronary arteries. Int J Cardiol 1999;68:275-280.

11 Erem C, Savaskan H, Cagilci F, Deger O, Karahan $\mathrm{C}$, Korkmaz H, Ulusoy S, Sonmez M, Cihanyurdu N, Telatar M: Plasma endothelin1 levels in patients with type II diabetes mellitus. Diabetes Res 1996;31:105-111.

12 Standl E, Balletshoffer B, Dahl B, Weichenhain B, Stiegler H, Hormann A, Holee R: Predictors of 10-year macrovascular and overall mortality in patients with NIDDM: The Munich General Practitioner Project. Diabetologia 1996;39: 1540-1545.

13 Kario K, Matsuo T, Kobayashi M, Matsuo M, Sakata T, Miyata T: Activation of tissue factorinduced coagulation and endothelial cell dysfunction in non-insulin-dependent diabetic patients with microalbuminuria. Arterioscler Thromb Vasc Biol 1995;15:1114-1120.
14 Kvasnicka J, Skrha J, Perusicova J, Kvasnicka T, Markova M, Umlaufova A, Pecen L: Haemostasis, cytoadhesive molecules (sE-selectin and sICAM-1) and inflammatory markers in non-insulin-dependent diabetes mellitus. $\mathrm{Sb}$ Lek 1998;99:97-101.

15 Gram J, Jespersen J: Induction and possible role of fibrinolysis in diabetes mellitus. Semin Thromb Hemost 1991;17:412-416.

16 Takahashi H, Tsuda A, Tatewaki W, Wada K, Niwano H, Shibata A: Activation of blood coagulation and fibrinolysis in diabetes mellitus: Evaluation by plasma levels of thrombinantithrombin III complex and plasmin-alpha 2-plasmin inhibitor complex. Thromb Res 1989;55:727-735.

17 Mansfield MW, Grant PJ: Fibrinolysis and diabetic retinopathy in NIDDM. Diabetes Care 1995; 18:1577-1581.

18 Hung Ho C, Shing Jap T: Fibrinolytic activity in Chinese patients with diabetes or hyperlipidemia in comparison with healthy controls. Thromb Haemost 1991;65:3-6.

19 Sharma SC: Platelet adhesiveness, plasma fibrinogen, and fibrinolytic activity in juvenileonset and maturity-onset diabetes mellitus. J Clin Pathol 1981;34:501-503.

$\checkmark 20$ Juhan-Vague I, Vague P, Poisson C, Aillaud MF, Mendez C, Collen D: Effect of 24 hours of normoglycemia on tissue-type plasminogen activator plasma levels in insulin-dependent diabetes. Thromb Haemost 1984;51:97-98. 
-21 Takada Y, Urano T, Waranabe I, Taminato A, Yoshimi T, Takada A: Changes in fibrinolytic parameters in male patients with type 2 (noninsulin-dependent) diabetes mellitus. Thromb Res 1993;71:405-415.

22 Vicari AM, D’Angelo V, Testa S, Comi G, Galardi G, Orsi E, D'Angelo A: Normal tissue plasminogen activator and plasminogen activator inhibitor activity in plasma from patients with type 1 diabetes mellitus. Horm Metab Res 1992;24:516-519.

$\checkmark 23$ Small M, Kluft C, MacCuish AC, Lowe GDO: Tissue plasminogen activator inhibition in diabetes mellitus. Diabetes Care 1989;12:655658.

-24 Maiello M, Boeri D, Podesta F, Cagliero E, Vichi M, Odetti P, Adezati L, Lorenzi M: Increased expression of tissue plasminogen activator and its inhibitor and reduced fibrinolytic potential of human endothelial cells cultured in elevated glucose. Diabetes 1992;41:10091015.

-25 Torr-Brown SR, Sobel BE: Plasminogen activator is elevated in plasma and diminished in platelets in patients with diabetes mellitus. Thromb Res 1994;75:473-477.

-26 Knobl P, Schernthaner G, Schnack C, et al: Thrombogenic factors are related to urinary albumin excretion rate in type 1 (insulindependent) and type 2 (non-insulin-dependent) diabetic patients. Diabetologia 1993;36:10451050 .

$\checkmark 27$ Gabazza EC, Takeya H, Deguchi H, Sumida Y, Taguchi O, Murata K, Nakatani K, Yano Y, Mohri M, Sata M, Shima T, Nishioka J, Suzuki $\mathrm{K}$ : Protein $\mathrm{C}$ activation in NIDDM patients. Diabetologia 1996;39:1455-1461.

-28 Wieczorek I, Pell ACH, McIver B, MacGregor IR, Ludlam CA, Frier BM: Coagulation and fibrinolytic systems in type 1 diabetes: Effects of venous occlusion and insulin-induced hypoglycemia. Clin Sci 1993;84:79-86.
29 Asakawa H, Tokunaga K, Kawakami F: Elevation of fibrinogen and thrombin-antithrombin III complex levels of type 2 diabetes mellitus patients with retinopathy and nephropathy. $\mathrm{J}$ Diabetes Complications 2000;14:121-126.

30 Aso Y, Matsumoto S, Fujiwara Y, Tayama K, Takebayashi K, Inukai T, Takemura Y: Impaired fibrinolytic compensation for hypercoagulability in obese patients with type 2 diabetes: Association with increased plasminogen activator inhibitor-1. Metabolism 2002;51: 471-476.

31 Sower JR, Lester MA: Diabetes and cardiovascular disease. Diabetes Care 1999;22:C14C20.

32 Hu J, Wei W, Din G, Yuan L, Liu Z: Variations and clinical significance of coagulation and fibrinolysis parameters in patients with diabetes mellitus. J Tongji Med Univ 1998;18: 233-235.

33 Ceriello A: Coagulation activation in diabetes mellitus: The role of hyperglycaemia and therapeutic prospects. Diabetologia 1993;36:11191125.

34 Ceriello A, Giugliano D, Quatraro A, et al: Induced hyperglycaemia alters antithrombin III activity but not its plasma concentration in healthy normal subjects. Diabetes 1987;36: 320-323.

35 Okazaki M, Zhang H, Tsuji M, Morio Y, Oguchi $\mathrm{K}$ : Blood coagulability and fibrinolysis in streptozotocin-induced diabetic rats. J Atheroscler Thromb 1997;4:27-33.

36 Vukovich TC, Schernthaner G: Decreased protein $\mathrm{C}$ levels in patients with insulin-dependent type 1 diabetes mellitus. Diabetes 1986;35: 617-619.
37 Jensen $\mathrm{T}$, Knudsen JB, Rasmussen BF, Deckert T: Features of endothelial dysfunction in early diabetic nephropathy. Lancet $1989 ; \mathrm{i}$ : 461-463.

38 Van den Berg M, Boers GH, Franken DG, Blom HJ, Van Kamp GJ, Jakobs C, Rauwerda JA, Kluft C, Stehouwert CD: Hyperhomocysteinaemia and endothelial dysfunction in young patients with peripheral arterial occlusive disease. Eur J Clin Invest 1995;25:176181.

39 Stehouwer CDA, Nuatta JJP, Zeldenrush GC, Hackeng WHL, Donker AJM, den Ottolander GJH: Urinary albumin excretion, cardiovascular disease, and endothelial dysfunction in noninsulin-dependent diabetes mellitus. Lancet 1992;340:319-323.

40 King GL, Banskota NK: Mechanisms of diabetic microvascular complications; in Kahn CR, Weir GC (eds): Joslin's Diabetes Mellitus, ed 13. Philadelphia, Lea \& Febiger, 1994, pp 631-647.

41 Gonzales J, Colwell JA, Sarki KE, et al: Effect of metabolic control with insulin on plasma von Willebrand factor activity (VIIIR:WF) in diabetes mellitus. Thromb Res 1980;17:261266.

42 Juhan-Vague I, Roul C, Alessi MC, Ardissone JP, Heim M, Vague P: Increased plasminogen activator inhibitor activity in non insulin dependent diabetic patients-relationship with plasma insulin. Thromb Haemost 1989;61: 370-373.

43 Auwerx J, Bouillon R, Collen D, Geboers J: Tissue-type plasminogen activator antigen and plasminogen activator inhibitor in diabetes mellitus. Arteriosclerosis 1988;8:68-72.

44 Aso Y, Fujiwara Y, Tayama K, Takebayashi K, Inukai T, Takemura Y: Relationship between soluble thrombomodulin in plasma and coagulation or fibrinolysis in type 2 diabetes. Clin Chim Acta 2000;301:135-145. 